\title{
MOST SUBMILLIMETER GALAXIES ARE MAJOR MERGERS
}

\author{
H. Engel ${ }^{1}$, L. J. Tacconi ${ }^{1}$, R. I. Davies ${ }^{1}$, R. Neri ${ }^{2}$, I. Smail ${ }^{3}$, S. C. Chapman ${ }^{4}$, R. Genzel ${ }^{1}$, P. Cox ${ }^{2}$, T. R. Greve ${ }^{5}$, \\ R. J. IVISON ${ }^{6,7}$, A. BLAIN ${ }^{8}$, F. BERTOLDI ${ }^{9}$, AND A. OMONT ${ }^{10}$ \\ ${ }^{1}$ Max-Planck-Institut für extraterrestrische Physik, Postfach 1312, 85741 Garching, Germany \\ ${ }^{2}$ Institut de Radio Astronomie Millimetrique (IRAM), St. Martin d'Heres, France \\ ${ }^{3}$ Institute for Computational Cosmology, Durham University, Durham DH1 3LE, UK \\ ${ }^{4}$ Institute of Astronomy, University of Cambridge, Madingley Road, Cambridge CB3 OHA, UK \\ ${ }^{5}$ Dark Cosmology Centre, Niels Bohr Institute, University of Copenhagen, Juliane Maries Vej 30, DK-2100 Copenhagen Ø, Denmark \\ ${ }^{6}$ UK Astronomy Technology Centre, Royal Observatory, Blackford Hill, Edinburgh EH9 3HJ, UK \\ ${ }^{7}$ Institute for Astronomy, University of Edinburgh, Blackford Hill, Edinburgh EH9 3HJ, UK \\ ${ }^{8}$ Astronomy 105-24, California Institute of Technology, Pasadena, CA 91125, USA \\ ${ }^{9}$ Argelander-Institut für Astronomie, Auf dem Hügel 71, 53121 Bonn, Germany \\ ${ }^{10}$ CNRS and Institut d'Astrophysique de Paris, 98 bis boulevard Arago, 75014 Paris, France \\ Received 2010 April 12; accepted 2010 September 10; published 2010 November 1
}

\begin{abstract}
We analyze subarcsecond resolution interferometric CO line data for 12 submillimeter-luminous $\left(S_{850 \mu \mathrm{m}} \geqslant 5 \mathrm{mJy}\right)$ galaxies with redshifts between 1 and 3, presenting new data for 4 of them. Morphologically and kinematically, most of the 12 systems appear to be major mergers. Five of them are well-resolved binary systems, and seven are compact or poorly resolved. Of the four binary systems for which mass measurements for both separate components can be made, all have mass ratios of 1:3 or closer. Furthermore, comparison of the ratio of compact to binary systems with that observed in local ULIRGs indicates that at least a significant fraction of the compact submillimeter-luminous galaxies (SMGs) must also be late-stage mergers. In addition, the dynamical and gas masses we derive are most consistent with the lower end of the range of stellar masses published for these systems, favoring cosmological models in which SMGs result from mergers. These results all point to the same conclusion that most of the bright SMGs with $L_{\mathrm{IR}} \gtrsim 5 \times 10^{12} L_{\odot}$ are likely major mergers.
\end{abstract}

Key words: cosmology: observations - galaxies: evolution - galaxies: formation - galaxies: high-redshift galaxies: kinematics and dynamics

Online-only material: color figures

\section{INTRODUCTION}

Surveys of dust-obscured galaxies at high redshifts carried out with SCUBA (Holland et al. 1999) and MAMBO (Kreysa et al. 1998) revealed a high-redshift $(z \sim 1-3.5)$ population of submillimeter-luminous $\left(S_{850 \mu \mathrm{m}} \geqslant 5 \mathrm{mJy}\right)$ galaxies (SMGs hereafter; see, e.g., Greve et al. 2004; Coppin et al. 2005; Chapman et al. 2005; Pope et al. 2005; Weiss et al. 2009). This population consists of dusty, gas-rich, very luminous $\left(\sim 10^{13} L_{\odot}\right)$, intensely star-forming $\left(\sim 10^{3} M_{\odot} \mathrm{yr}^{-1}\right)$ galaxies (e.g., Greve et al. 2005; Chapman et al. 2005). The importance of these SMGs is underlined by studies of the extragalactic far-IR/ submillimeter background, which shows that about half of the cosmic energy density originates in distant, dusty starbursts and active galactic nuclei (AGNs; Puget et al. 1996; Pei et al. 1999), with ULIRGs contributing 20\% (Magnelli et al. 2010). NearIR (Swinbank et al. 2004), optical and X-ray (Alexander et al. 2005), and mid-IR (Lutz et al. 2005; Valiante et al. 2007; Pope et al. 2008; Menéndez-Delmestre et al. 2009) spectroscopic works have shown that starbursts are the primary power source, with AGNs present in a large fraction but not making a dominant energetic contribution.

A key question is what triggers the extraordinary luminosities of these galaxies. A number of authors have suggested that at least some SMGs may be interacting or merging systems, based primarily on irregular or complex morphologies seen in restframe UV Hubble Space Telescope (HST) images (Chapman et al. 2003, 2004; Smail et al. 2004; Swinbank et al. 2006). However, two caveats need to be kept in mind regarding these results. UV emission is strongly affected by extinction, and hence UV images may not reliably track the underlying galaxy. This is demonstrated by the radio observations carried out by Chapman et al. (2004) and Biggs \& Ivison (2008; a key result of these studies was that the star formation in SMGs is more extended than in local ULIRGs, an issue which we discuss in more detail later), which show morphological differences between radio and UV emission. Furthermore, morphological studies alone may be misleading. High-redshift galaxies are clumpy and irregular, which may either be the result of ongoing mergers (Lotz et al. 2006; Conselice et al. 2008), or the consequence of clumpy star formation in a kinematically more coherent structure such as an underlying rotating disk (FörsterSchreiber et al. 2006, 2009; Genzel et al. 2006; Elmegreen et al. 2007), or strong and structured extinction. Ivison et al. (2002, 2007) were able to show that SMGs have an increased likelihood of having close neighbors, suggestive of physical associations. Swinbank et al. (2006) obtained integral field near-infrared spectroscopy data of six SMGs, which showed a significant fraction consisting of kinematically distinct subcomponents, interpreted as an indication of merging. However, as with the HST data discussed above and as also pointed out by Swinbank et al. (2010), these rest-frame optical and UV data suffer from extinction limitations and potential biases due to outflows. Ideally, we need spatially resolved maps and kinematics of a tracer that is not affected by dust extinction to properly assess morphologies and kinematics. Using highresolution millimeter $\mathrm{CO}$ line observations, Tacconi et al. (2006, 2008) find SMGs to display either double/multiple 


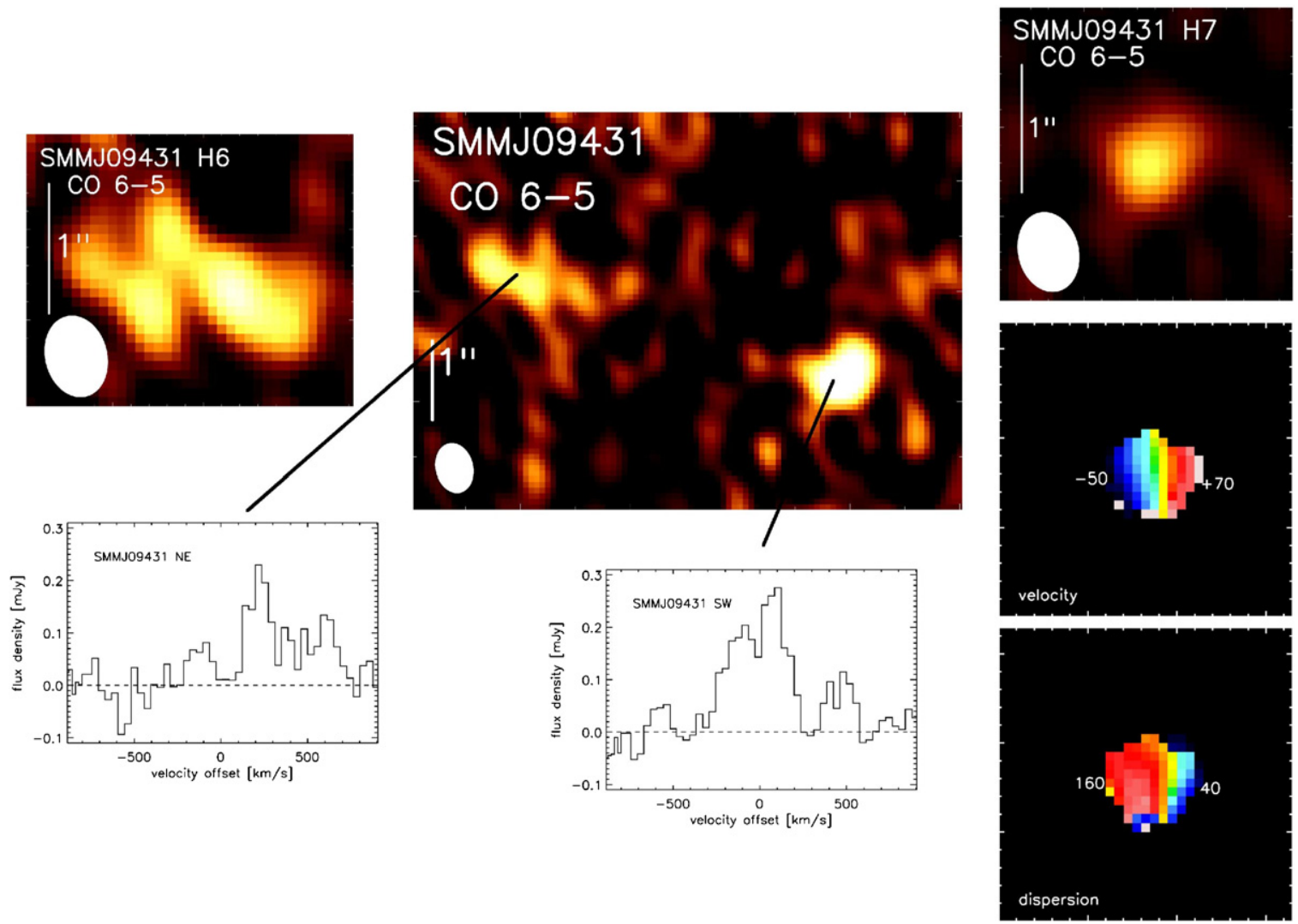

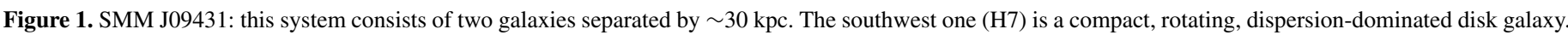

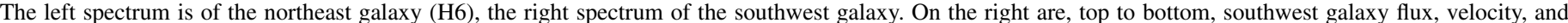

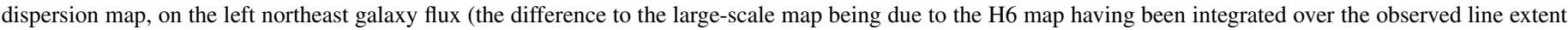

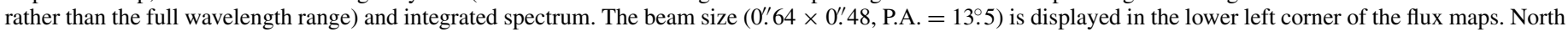
is up and east is to the left. Scale bars denote $1^{\prime \prime}$.

(A color version of this figure is available in the online journal.)

morphologies with complex gas motions indicative of ongoing mergers, or to be extremely dense, compact, rotating disks. The high gas and matter densities of the latter argue for them to be end-stage major mergers. However, the spatial resolution achievable with current facilities is insufficient to definitively distinguish between coalesced major mergers and isolated disk galaxies. At present, it is thus still unclear whether SMGs form a homogeneous class of galaxies, or whether their high luminosities can be achieved through a variety of pathways. Illustrative of this ongoing discussion are the debates about the nature of two of the first SMGs to be discovered: SMM J02399-136 and SMM J14011+0252 (Ivison et al. 1998, 2000; Smail et al. 2002; Genzel et al. 2003; Ivison et al. 2010; Downes \& Solomon 2003; Smail et al. 2005).

The aim of this paper is to critically examine the hypothesis that SMGs are mergers. We use observations of the CO line, which is unaffected by extinction or outflows, to trace the molecular gas, and combine both morphological and kinematic aspects in the analysis. We present new data for four SMGs. One of our sources has not been observed in CO before (SMM J105141); for the three other systems these new observations provide data of significantly increased resolution. This brings the number of SMGs observed to subarcsecond resolution from 8 to 12 and thus provides a significant increase in the total sample of highresolution CO SMG observations. We complement these new data with previously published high-resolution $\mathrm{CO}$ data for eight other SMGs (Greve et al. 2005; Hainline et al. 2006; Tacconi et al. 2006, 2008; Ivison et al. 2010; Bothwell et al. 2010), re- sulting in a much-improved statistical basis compared to, e.g., Tacconi et al. (2006, 2008). Our work is distinct from these preceding works, the aim of which was a general characterization of the SMG population, not only in regard to sample size, but also in terms of objective. Here, we tackle a specific, heretofore unsolved key issue-namely, whether or not SMGs are major mergers.

In Section 2, we detail our observations and data reduction. In Section 3, we explain the analyses employed, and present results for individual systems. We then investigate merger mass ratios, and sizes of our sample galaxies. In Section 4, we test stellar mass estimates from the literature, and discuss the implication of our results for cosmological models. We then bring these results together to discuss the trigger mechanism for the exceptional luminosities of SMGs, before concluding in Section 5.

\section{OBSERVATIONS AND DATA REDUCTION}

The observations of four new SMGs were carried out in winter $2007 / 2008$ with the IRAM Plateau de Bure interferometer, which consists of six $15 \mathrm{~m}$ diameter telescopes (Guilloteau et al. 1992). We observed the four sources, SMM J09431, SMM J105141, SMM J131201, and HDF 169, under excellent weather conditions in the extended A configuration, which has baselines up to $760 \mathrm{~m}$. The resulting spatial resolutions ranged from 0'.4 to 0'.6 (see Figures 1-4). For the two $z \sim 3$ sources, SMM J09431 and SMM J131201, we observed the CO $J=$ $6-5$ line, which is redshifted into the $2 \mathrm{~mm}$ band. For the two 


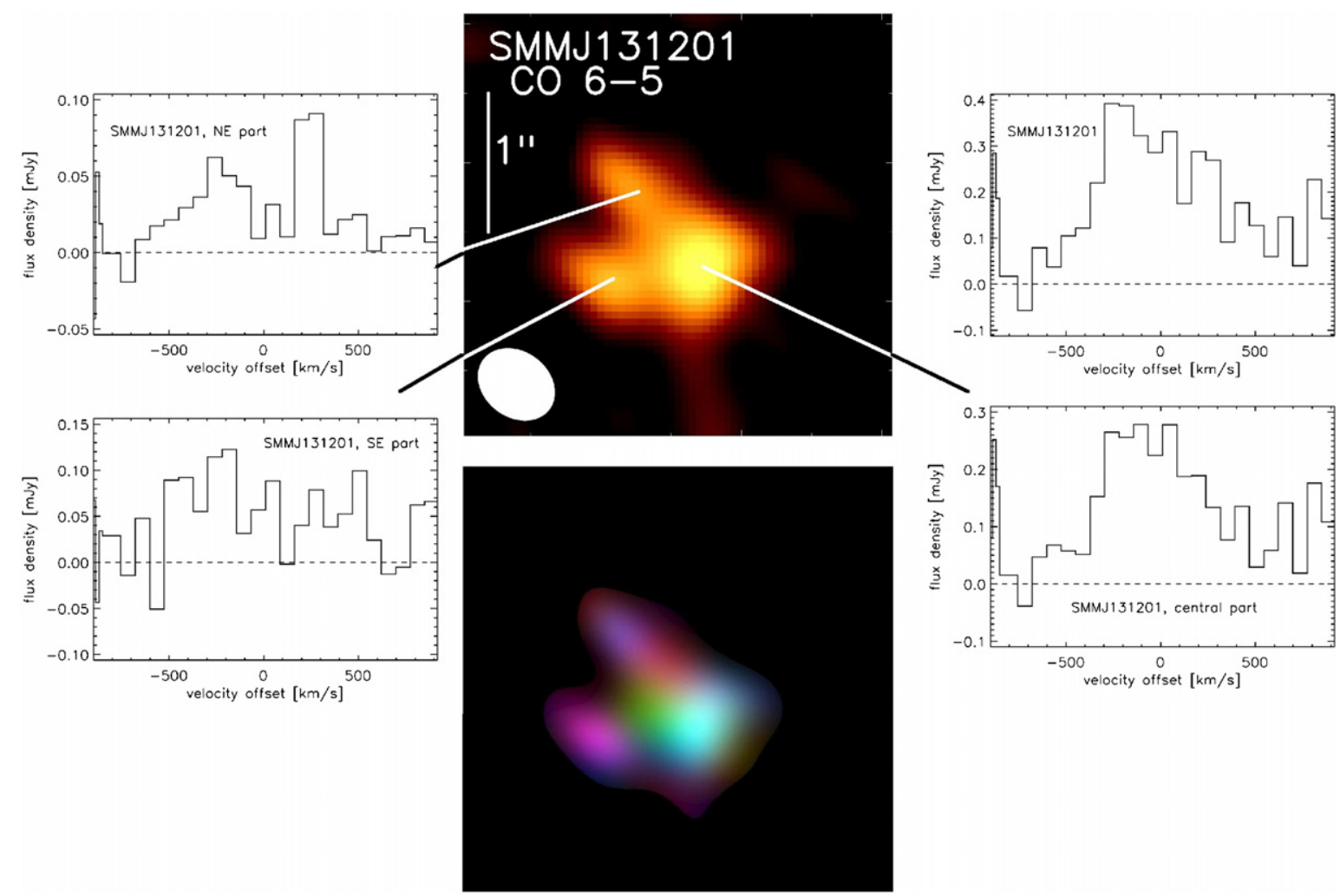

Figure 2. SMM J131201: this system displays a complex, irregular morphology; it most likely is an advanced pre-coalescence merger. The RGB image (mapping red, green, and blue parts of the spectrum) shows no indication of rotation. Spectra are, left: northeast (top) and southeast (bottom) arm, right: entire system (top), and central part (bottom). The beam size $\left(0^{\prime \prime} 59 \times 00^{\prime \prime} 47\right.$, P.A. $\left.=50.9\right)$ is displayed in the lower left corner of the flux maps. North is up and east is to the left. Scale bar denotes $1^{\prime \prime}$.

(A color version of this figure is available in the online journal.)

sources at $z \sim 1.2$, SMM J105141 and HDF 169, we observed the CO $J=4-3$ line in the $1 \mathrm{~mm}$ band. The observations were all made with the new generation, dual polarization receivers, with system temperatures of $\sim 150 \mathrm{~K}$ and $\sim 200 \mathrm{~K}$ (SSB) in the $2 \mathrm{~mm}$ and $1 \mathrm{~mm}$ bands, respectively. The full available correlator capacity was configured to cover $1 \mathrm{GHz}$ in each polarization, which adequately covers the broad $\mathrm{CO}$ lines seen in SMGs (Genzel et al. 2003; Neri et al. 2003; Greve et al. 2005). The total on-source integration times were $9.3 \mathrm{hr}$ for SMM J09431, 12.7 hr for SMM J105141, 14.4 hr for HDF 169, and $20.1 \mathrm{hr}$ for SMM J131204.

We calibrated the data sets using the CLIC program in the IRAM GILDAS package. Passband calibration used one or more bright quasars. Every 20 minutes, we alternated source observations with a bright quasar calibrator within $15^{\circ}$ of the source. Our absolute positional accuracies are \pm 0 '. 2 or better. The absolute flux scale was calibrated on MWC 349 (1.35 Jy at $\sim 156 \mathrm{GHz}$ and $1.6 \mathrm{Jy}$ at $\sim 208 \mathrm{GHz}$ ). After flagging bad- and high-phase noise data, we created data cubes in the MAPPING environment of GILDAS. Final maps were cleaned with the CLARK version of CLEAN implemented in GILDAS. The absolute flux scale is better than $\pm 20 \%$.

\section{RESULTS}

Integrated spectra, integrated line flux maps, position-velocity $(\mathrm{P}-\mathrm{V})$ diagrams, and velocity and velocity dispersion maps of the individual systems are displayed in Figures 1-4. Measured and derived source properties are listed in Tables 1-3.

\subsection{Analysis}

Our analyses generally follow the procedures of Tacconi et al. (2006, 2008); for the computation of cold gas masses, we use the approach of Tacconi et al. (2010). Flux maps were produced by integrating the line emission, determining the line extent via visual inspection of spatially integrated spectra. Fluxes are extracted from the line cubes by integrating over the area with significant emission. Given that the compact galaxies comprising a significant fraction of our sample here are in most cases only marginally resolved with the $\sim 0^{\prime \prime} .5-1^{\prime \prime}$ spatial resolution available, we determine source sizes by fitting circular Gaussians to the systems in the $u v$-plane using the task UVFIT in the IRAM data reduction package GILDAS, and taking the Gaussian FWHM $_{\mathrm{uv}}$ to be the best estimate of the source's diameter. In the two individual galaxies of the four new systems where a Gaussian fit was not possible (most likely due to insufficient signal to noise), the beam FWHM was used as an upper limit to the source diameter. We note that these size measurements may slightly underestimate the actual size of the galaxies, since there may be faint extended flux below our sensitivity limit. We do not assign a formal uncertainty to this, since, if such a putative faint emission region exists, we do not know anything about its size or morphology. However, the uncertainties due to this are unlikely to be significant.

Where possible, velocity and velocity dispersion maps were derived by fitting Gaussians to the line profiles. If the spectral signal to noise did not permit this, we produced moment maps 

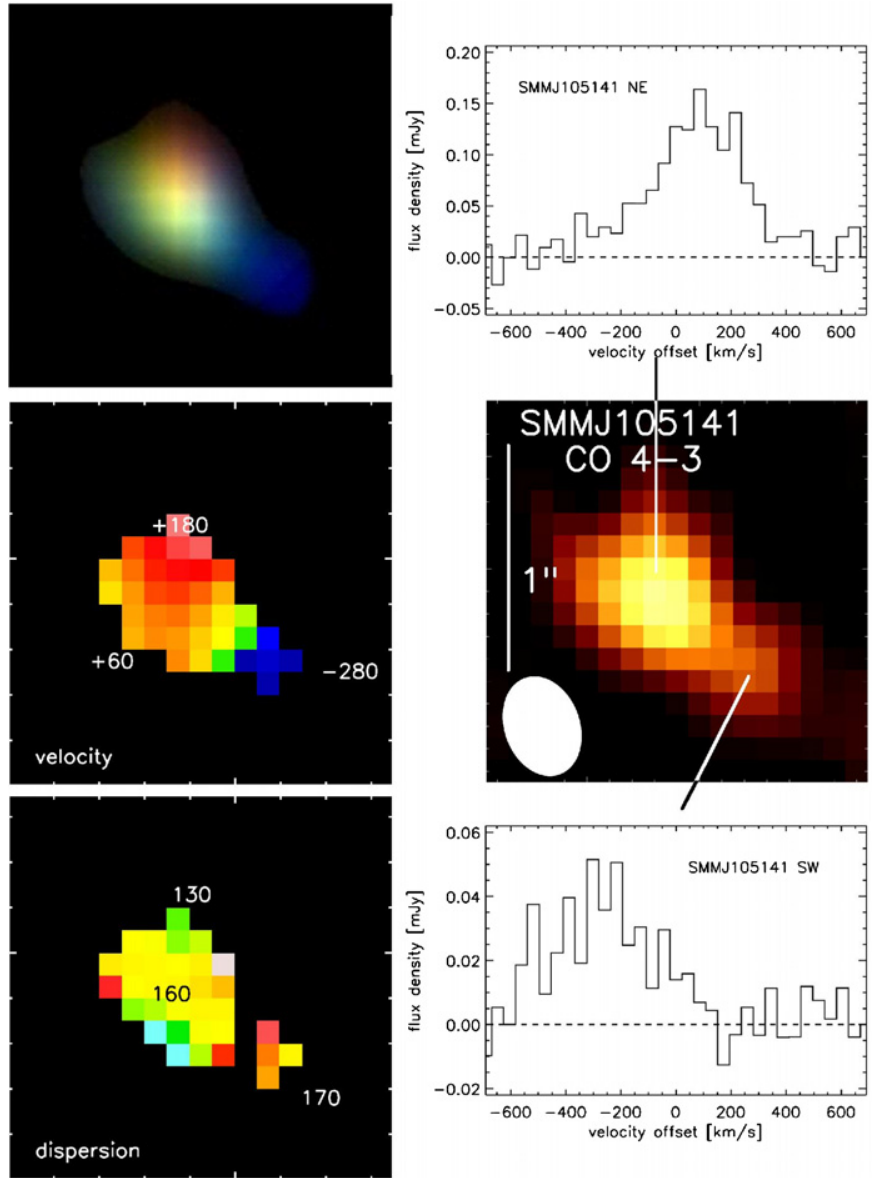

Figure 3. SMM J105141: as the RGB map (top; mapping red, green, and blue parts of the spectrum) clearly shows, this system consists of two galaxies that are separated in velocity by $\sim 400 \mathrm{~km} \mathrm{~s}^{-1}$. Top right spectrum is of "main" component (northeast galaxy), bottom right spectrum of the blueshifted component (southwest galaxy). As can be seen from the velocity and dispersion maps (left, middle and bottom), the northeast galaxy is a dispersion-dominated disk galaxy. The beam size $\left(0^{\prime \prime} 46 \times 0.34\right.$, P.A. $\left.=28^{\circ} .7\right)$ is displayed in the lower left corner of the flux maps. North is up and east is to the left. Scale bar denotes $1^{\prime \prime}$.

(A color version of this figure is available in the online journal.)

of different velocity ranges, which were then overlaid as RGB images.

Since the dispersions of our galaxies are comparable to or larger than the rotational velocities, dynamical masses have been derived using the "isotropic virial estimator" (e.g., Spitzer 1987; Tacconi et al. 2008; Förster-Schreiber et al. 2009) given in $M_{\odot}$ by

$$
M_{\mathrm{dyn}, \mathrm{vir}}=2.8 \times 10^{5} \Delta v_{\mathrm{FWHM}}^{2} R_{1 / 2} \text {, }
$$

where $\Delta v_{\text {FWHM }}\left(\mathrm{km} \mathrm{s}^{-1}\right)$ is the integrated line FWHM and $R_{1 / 2}$ (kpc) is the half-light radius (the radius within which half the total flux is emitted, here assumed to be equivalent to the Gaussian HWHM measured in the $u v$-plane). We note that the scaling factor appropriate for a rotating disk at an average inclination is a factor of $\sim 1.5$ smaller (Bothwell et al. 2010), i.e., if the above formula were applied to a disk galaxy, it would somewhat overestimate the dynamical mass. However, Cappellari et al. (2009) find that this estimator agrees well with masses derived from more detailed Jeans modeling for a sample of massive $z \sim 2$ early-type galaxies.
In order to derive molecular gas masses, we convert the measured line fluxes to $\mathrm{CO}(1-0)$ fluxes (in $\mathrm{Jy} \mathrm{km} \mathrm{s}^{-1}$ ) using the results of SMG spectral energy distribution (SED) modeling (Figure 3 of Weiss 2007; yielding $S_{\mathrm{CO}(6-5)} / S_{\mathrm{CO}(1-0)}=$ $13, S_{\mathrm{CO}(4-3) / \mathrm{CO}(1-0)}=10, S_{\mathrm{CO}(3-2)} / S_{\mathrm{CO}(1-0)}=7, S_{\mathrm{CO}(2-1)} /$ $S_{\mathrm{CO}(1-0)}=3$; the same numbers were also used by Bothwell et al. 2010 and Casey et al. 2009b). We assign an uncertainty of $30 \%$ to these conversions based on the differences between the individual SED models of Weiss (2007); in the four cases where we have flux measurements from two different transitions (Table 1), we find the flux ratios to be consistent with these conversion factors. We do not calculate gas masses based on $\mathrm{CO}(7-6)$ fluxes, since the conversion factor is uncertain for this transition. We then calculate the $\mathrm{CO}(1-0)$ line luminosities (in $\mathrm{K} \mathrm{km} \mathrm{s}^{-1} \mathrm{pc}^{2}$ ) using Equation (3) of Solomon et al. (1997):

$$
L_{\mathrm{CO}(1-0)}^{\prime}=3.25 \times 10^{7} S_{\mathrm{CO}(1-0)} \Delta V v_{\mathrm{CO}(1-0), \text { rest }}^{-2} D_{L}^{2}(1+z)^{-1},
$$

with $S_{\mathrm{CO}} \Delta V$ in $\mathrm{Jy} \mathrm{km} \mathrm{s}{ }^{-1}, v_{\mathrm{CO}(1-0) \text {, rest }}$ in $\mathrm{GHz}$, and $D_{L}$ in Mpc. The $\mathrm{CO}(1-0)$ luminosities are then used to estimate the total gas mass via their Equation (19):

$$
M_{\mathrm{H}_{2}}=\alpha L_{\mathrm{CO}(1-0)}^{\prime}
$$

using $\alpha=1 M_{\odot} / K \mathrm{~km} \mathrm{~s}^{-1} \mathrm{pc}^{2}$ as measured for ULIRGs (Downes \& Solomon 1998, for a discussion see also Genzel et al. 2010). For an in-depth discussion, we refer the reader to Appendix A of Tacconi et al. (2008); we would like to stress that our choice of $\alpha$ is motivated by the surface densities of SMGs (cf. Table 10 of Tacconi et al. 2008) rather than any a priori assumption about a similarity between local ULIRGs and SMGs. Our choice of conversion factor is here further supported by the fact that if for our SMGs the Milky Way conversion factor was adopted, in all but one case would the gas mass, rather implausibly, be equal to or larger than the dynamical mass. Finally, we apply a factor 1.36 correction to account for interstellar helium. We note that since the fluxes were measured in fixed apertures, and also that some flux may be resolved out or be below our detection limit, the gas mass is likely to be somewhat larger than our estimates.

\subsection{Results for Individual Objects}

The detailed analyses of the molecular CO line emission in this section reveal morphological and kinematic evidence that at least 8 of the 12 SMGs studied in this paper are ongoing mergers, and the remaining 4 are either disk galaxies or coalesced, latestage mergers.

\subsubsection{New Observations}

SMM J09431 $(z=3.35)$. A review of previous observations is given by Tacconi et al. (2006), who presented $\mathrm{CO}(4-3)$ data. The system consists of two galaxies, H6 and H7, separated by $\sim 30 \mathrm{kpc}$ in projection, and thus are physically related, and perhaps in an early stage of merging. Our new $\mathrm{CO}(6-5)$ data show a clear line spectrum for the southwest galaxy $\mathrm{H} 7$, with a global velocity gradient from -50 to $+70 \mathrm{~km} \mathrm{~s}^{-1}$, probably indicative of rotation. Förster-Schreiber et al. (2009) find that the commonly adopted boundary between "dispersion-dominated" and "rotation-dominated" systems of $v_{\text {rot }} \sim \sigma_{0}$ (where $v_{\text {rot }}$ is the circular/orbital velocity and $\sigma_{0}$ is the intrinsic velocity dispersion) translates into $\sigma_{\text {int }} / v_{\text {obs }} \sim 1.25$ (where $v_{\text {obs }}$ is the observed line-of-sight rotation and $\sigma_{\text {int }}$ is the integrated line FWHM divided by 2.355$)$. $\mathrm{H} 7$ has $\sigma_{\text {int }} / v_{\text {obs }}>1.5$ nearly everywhere, i.e., 

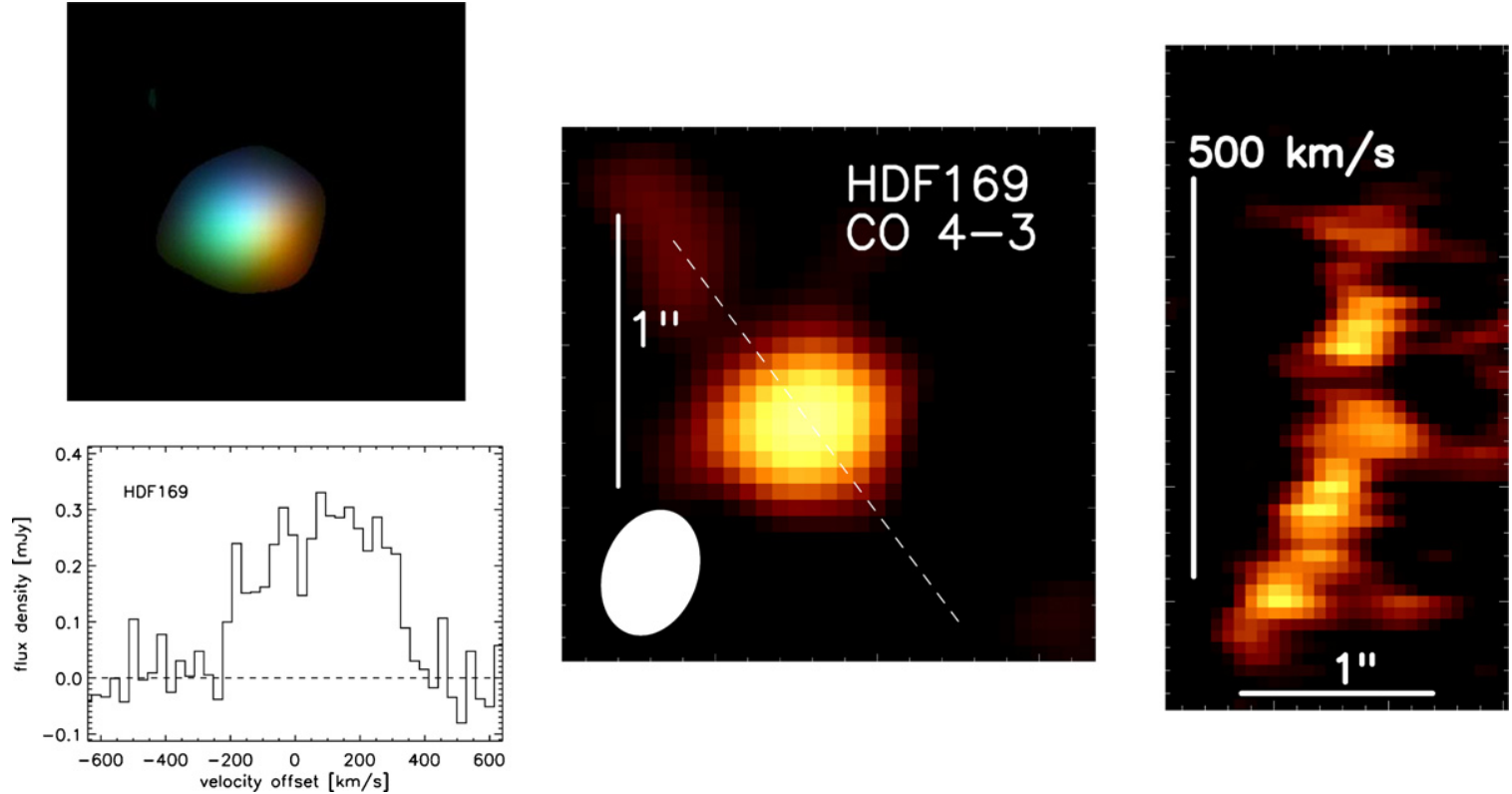

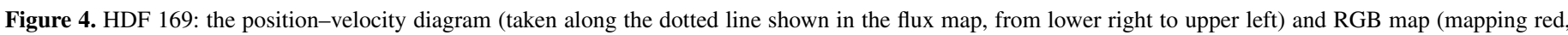




North is up and east is to the left. Scale bar denotes $1^{\prime \prime}$.

(A color version of this figure is available in the online journal.)

it appears dispersion dominated. For the northeast galaxy H6, we detect a $4 \sigma$ line shifted about $+210 \mathrm{~km} \mathrm{~s}^{-1}$ relative to $\mathrm{H} 7$. Its width is $180 \pm 65 \mathrm{~km} \mathrm{~s}^{-1}$. There may also be broader and more redshifted emission extended over a larger region around H6 and toward H7, but at low significance. When deriving gas masses, the measured fluxes and sizes are corrected by a factor of 1.3 for gravitational lensing effects, using the model of Cowie et al. (2002).

SMM J131201 $(z=3.41)$. This system shows complex spatial and velocity distributions. There is no evidence of ordered rotation, although the extensions to the east and northeast are redshifted with respect to the emission peak. The substructures, which may be clumps, outflows, or tidal tails, and the overall lack of rotation most likely point toward an advanced, precoalescence merger stage. This conclusion is also drawn by Hainline (2008).

SMM J105141 $(z=1.21)$. There are two galaxies separated in velocity by $\sim 400 \mathrm{~km} \mathrm{~s}^{-1}$ and spatially by $\sim 5 \mathrm{kpc}$, with no indications of disturbances. The southwest galaxy is about a factor 3 less luminous than its companion, but nearly equal in dynamical mass. The line profiles are well resolved. The velocity field of the northeast system is indicative of ordered rotation, with velocities ranging from 60 to $180 \mathrm{~km} \mathrm{~s}^{-1}$. Again, we find that generally $\sigma_{\text {int }} / v_{\text {obs }}>1.5$. It is likely that we are seeing two galaxies engaged in the early stages of merging, appearing close only in projection.

$H D F 169$ (SMM J123634+6212, $z=1.22$ ). The integrated spectrum of this compact galaxy is double-peaked, and its $\mathrm{P}-\mathrm{V}$ diagram resembles a rotation curve, both indicative of a compact rotating system. It has a mass surface density of $\approx 10^{3.3} M_{\odot} \mathrm{pc}^{-2}$. Tacconi et al. (2008) argued that the very high volume and surface densities characterizing compact systems such as this could only plausibly be achieved during the final stages of a major merger. However, we feel that this argument alone is insufficient to judge with any degree of certainty whether this system is a quiescent disk galaxy or a late-stage merger.

\subsubsection{Re-analysis of Existing Observations and Published Results}

We have re-analyzed previous observations of HDF 242 (SMM J123707+614, $z=2.49$; Tacconi et al. 2006) and N2850.4 (SMM J163650+4057 and SMM J16368+4057, $z=$ 2.38; Tacconi et al. 2006) using the methods described in Section 3.1 (see Table 1). We also complement our data with published results from $\mathrm{CO}$ line observations by Tacconi et al. (2006, 2008) and Bothwell et al. (2010) for five other systems; HDF 76 (SMM J123549+6215, $z=$ 2.20), N2850.2 (SMM J163658+4105, SMM J16370+4105, and SMM J16366+4105, $z=2.45)$, SMM J044307+0210 (SMM J04431+0210, $z=2.51$ ), HDF 132 (SMM J123618.33+ $621550.5, z+2.00)$, and Lockman 38 (SMM J105307+572431.4, $z=1.52)$, and where possible, re-calculating derived quantities to bring them into a consistent methodological framework. Finally, we include results from new multi-wavelength observations of SMM J02399-0136 (Ivison et al. 2010). The published results on the kinematics and morphologies of these systems are summarized below.

SMM J044307+0210. This lensed galaxy appears compact and has a double-peaked line profile with a FWHM of $\sim 350 \mathrm{~km} \mathrm{~s}^{-1}$.

$H D F 242$. A wide binary with a separation of $\sim 22 \mathrm{kpc}$.

N2850.4. The $\mathrm{CO}(3-2)$ data show a double-peaked line profile and a separation of $\sim 3.3 \mathrm{kpc}$ between the peaks of the red and blue channel maps. The integrated emission in the higher resolution $\mathrm{CO}(7-6)$ data directly shows two peaks separated by $\sim 3.7 \mathrm{kpc}$. This system is therefore interpreted as a close binary. We infer a mass ratio of 0.68 from the flux ratios of the two components.

$H D F$ 76, N2850.2, and HDF 132. All are compact sources with double-peaked line profiles. They could be either coalesced major mergers, or massive disk galaxies. They have extremely high-mass surface densities of $10^{3.5}, 10^{4.5}$, and $10^{4.0} M_{\odot} \mathrm{pc}^{-2}$, respectively (Tacconi et al. 2008; Bothwell et al. 2010), at least 
Table 1

Measured and Derived Quantities

\begin{tabular}{|c|c|c|c|c|c|c|}
\hline Galaxy & $\begin{array}{c}\text { Obs. CO } \\
\text { Transition } \\
\end{array}$ & $\begin{array}{c}S_{\mathrm{CO}} \Delta V \\
\left(\mathrm{Jy} \mathrm{km} \mathrm{s}^{-1}\right)\end{array}$ & $\begin{array}{l}\mathrm{HWHM}_{u v} \\
\quad(\mathrm{kpc})\end{array}$ & $\begin{array}{c}v_{\text {FWHM }} \\
\left(\mathrm{km} \mathrm{s}^{-1}\right)\end{array}$ & $\begin{array}{c}M_{\mathrm{gas}} \\
\left(10^{10} M_{\odot}\right)\end{array}$ & $\begin{array}{c}M_{\mathrm{dyn}} \\
\left(10^{10} M_{\odot}\right)\end{array}$ \\
\hline \multirow[t]{3}{*}{ SMM J09431, SW } & $6-5^{a}$ & $1.8 \pm 0.3$ & $1.13 \pm 0.23$ & $390 \pm 45$ & $7.10 \pm 2.44$ & $4.81 \pm 1.26$ \\
\hline & $4-3^{b}$ & $1.1 \pm 0.2$ & $1.85 \pm 0.60$ & $400 \pm 45$ & $5.64 \pm 1.98$ & $8.29 \pm 2.99$ \\
\hline & $1-0^{\mathrm{c}}$ & $<0.13$ & $\mathrm{n} / \mathrm{a}$ & $\mathrm{n} / \mathrm{a}$ & $<8.67$ & $\mathrm{n} / \mathrm{a}$ \\
\hline \multirow[t]{2}{*}{ SMM J09431, NE } & $6-5^{a}$ & $1.1 \pm 0.2$ & $\mathrm{n} / \mathrm{a}$ & $\mathrm{n} / \mathrm{a}$ & $4.34 \pm 1.53$ & $\mathrm{n} / \mathrm{a}$ \\
\hline & $4-3^{b}$ & $<0.4$ & $\mathrm{n} / \mathrm{a}$ & $\mathrm{n} / \mathrm{a}$ & $<2.05$ & $\mathrm{n} / \mathrm{a}$ \\
\hline \multirow[t]{3}{*}{ SMM J131201 } & $6-5^{a}$ & $2.54 \pm 0.1$ & $4.09 \pm 0.51$ & $911 \pm 140$ & $13.43 \pm 4.97$ & $95.04 \pm 23.81$ \\
\hline & $4-3^{d}$ & $1.7 \pm 0.3$ & $\mathrm{n} / \mathrm{a}$ & $530 \pm 50$ & $11.67 \pm 4.06$ & $\mathrm{n} / \mathrm{a}$ \\
\hline & $1-0^{\mathrm{c}}$ & $0.42 \pm 0.07$ & $\mathrm{n} / \mathrm{a}$ & $1040 \pm 190$ & $28.83 \pm 4.81$ & $\mathrm{n} / \mathrm{a}$ \\
\hline SMM J105141, SW & $4-3^{a}$ & $1.53 \pm 0.11$ & $<1.40$ & $436 \pm 50$ & $1.63 \pm 0.51$ & $<7.45$ \\
\hline SMM J105141, NE & $4-3^{a}$ & $5.87 \pm 0.19$ & $1.77 \pm 0.14$ & $383 \pm 50$ & $6.72 \pm 1.89$ & $7.27 \pm 1.46$ \\
\hline \multirow[t]{2}{*}{ HDF 169} & $4-3^{a}$ & $2.25 \pm 0.11$ & $2.15 \pm 0.24$ & $431 \pm 60$ & $2.45 \pm 0.75$ & $11.18 \pm 2.53$ \\
\hline & $2-1^{\mathrm{e}}$ & $3.45 \pm 0.93$ & $<16.5$ & $560 \pm 90$ & $12.47 \pm 5.03$ & $<145$ \\
\hline \multirow[t]{2}{*}{ HDF 76} & $6-5^{b, f}$ & $2.3 \pm 0.4$ & $0.95 \pm 0.4$ & $600 \pm 80$ & $5.76 \pm 2.00$ & $9.58 \pm 4.42$ \\
\hline & $3-2^{b, f}$ & $1.6 \pm 0.2$ & $<2.1$ & $600 \pm 50$ & $7.44 \pm 2.42$ & $<21.17$ \\
\hline HDF 242, SW & $3-2^{a, b, f}$ & $0.59 \pm 0.08$ & $2.51 \pm 0.86$ & $426 \pm 60$ & $3.41 \pm 1.13$ & $12.75 \pm 5.10$ \\
\hline HDF $242, \mathrm{NE}$ & $3-2^{a, b, f}$ & $0.32 \pm 0.07$ & $3.45 \pm 0.98$ & $394 \pm 60$ & $1.85 \pm 0.68$ & $15.00 \pm 5.35$ \\
\hline \multirow[t]{2}{*}{$\mathrm{N} 2850.2$} & $7-6^{\mathrm{b}, \mathrm{f}}$ & $3.3 \pm 0.5$ & $0.8 \pm 0.5$ & $790 \pm 50$ & $\mathrm{n} / \mathrm{a}$ & $13.98 \pm 8.83$ \\
\hline & $3-2^{b, d}$ & $1.8 \pm 0.2$ & $\mathrm{n} / \mathrm{a}$ & $800 \pm 50$ & $10.11 \pm 3.23$ & $\mathrm{n} / \mathrm{a}$ \\
\hline $\mathrm{N} 2850.4$, total & $3-2^{b, f}$ & $2.3 \pm 0.3$ & $2.48 \pm 1.24$ & $710 \pm 50$ & $12.28 \pm 4.02$ & $35.00 \pm 17.85$ \\
\hline $\mathrm{N} 2850.4, \mathrm{SW}$ & $7-6^{a, b, f}$ & $2.01 \pm 0.18$ & $0.54 \pm 0.37$ & $373 \pm 50$ & $\mathrm{n} / \mathrm{a}$ & $2.10 \pm 1.50$ \\
\hline $\mathrm{N} 2850.4, \mathrm{NE}$ & $7-6^{a, b, f}$ & $1.36 \pm 0.18$ & $<1.70$ & $139 \pm 50$ & $\mathrm{n} / \mathrm{a}$ & $<0.92$ \\
\hline SMM J044307 & $3-2^{b}$ & $1.4 \pm 0.2$ & $<3.26$ & $350 \pm 40$ & $8.20 \pm 2.73$ & $<11.18$ \\
\hline SMM J02399 & $1-0^{\mathrm{g}}$ & $\mathrm{n} / \mathrm{a}$ & $\mathrm{n} / \mathrm{a}$ & $\mathrm{n} / \mathrm{a}$ & $100 \pm 30$ & $\mathrm{n} / \mathrm{a}$ \\
\hline HDF 132 & $4-3^{h}$ & $1.27 \pm 0.14$ & 2.65 & $\mathrm{n} / \mathrm{a}$ & $3.49 \pm 1.12$ & $21.80 \pm 1.80$ \\
\hline Lockman 38 & $3-2^{\mathrm{h}}$ & $0.63 \pm 0.08$ & 4.75 & $\mathrm{n} / \mathrm{a}$ & $1.49 \pm 0.49$ & $6.20 \pm 1.10$ \\
\hline
\end{tabular}

Notes. Measured quantities for the 12 systems in our sample. New observations presented in this paper are in bold face. If a system consists of two galaxies, SW or NE indicates which galaxy is referred to. As outlined in the text, the HWHM of Gaussian fits to the source in the $u v$-plane were taken as best estimates of the intrinsic $R_{1 / 2}$, except for HDF 132 and Lockman 38, for which the half light radii as quoted by Bothwell et al. (2010) are given. The quoted values for dynamical masses were calculated using the "isotropic virial estimator" (see Section 3.1 for details), except for HDF 132 and Lockman 38, for which Bothwell et al. (2010) estimated the quoted masses using disk rotation models. Gas masses were calculated by converting the measured line flux to CO(1-0) flux using Figure 3 of Weiss (2007), assuming a ULIRG conversion factor $\alpha=1 M_{\odot} / \mathrm{K} \mathrm{km} \mathrm{s}^{-1} \mathrm{pc}^{2}$, and including a factor 1.36 correction for helium. For SMM J09431, measurements were corrected for gravitational lensing magnification of factor 1.3 (Cowie et al. 2002). If a source was unresolved, the beam HWHM was taken as an upper limit to $R_{1 / 2}$.

a This work.

b Tacconi et al. (2006).

c Hainline et al. (2006).

d Greve et al. (2005).

e Frayer et al. (2008).

f Tacconi et al. (2008)

g Ivison et al. (2010).

h Bothwell et al. (2010).

an order of magnitude larger than those measured in the UV/ optically selected SINS galaxies (Förster-Schreiber et al. 2006, 2009; Tacconi et al. 2008) and comparable to those measured in the central nuclear gas disk of Arp 220. Tacconi et al. (2008) argue, that to achieve this, the torques provided by a highly dissipative, major merger are necessary.

Lockman 38. Bothwell et al. (2010) interpreted this object as a very late-stage merger, having two kinematically distinct components that are not spatially resolved.

SMM J02399-0136. New multi-wavelength observations (Ivison et al. 2010) reveal that this system consists of at least two major components (most likely three, two of which host a $\mathrm{BH})$; it is therefore interpreted as an ongoing merger.

\subsection{Mass Ratios}

Ivison et al. (2002) first noted a preponderance of radio doubles among SMGs. With a larger sample, Ivison et al. (2007) were able to demonstrate that the likelihood of SMGs having companions within a few arcseconds is significantly larger than would be expected by chance. This overdensity suggests that at least a significant fraction of SMGs may currently be undergoing mergers. We are able to refine this picture substantially by investigating the mass ratios of the merging components. Five of the twelve SMGs in our sample consist of spatially separated interacting galaxies, and we are able to measure mass ratios for four of them (Table 2; SMM J09431, SMM J105141, HDF 242, $\mathrm{N} 2850.4$; no mass estimates for the individual components of SMM J02399 are published). In all cases, the mass ratios are consistent with being major (mass ratios of $1: 3$ or closer to unity) mergers. It must be kept in mind that if these are mergers between disk galaxies, the dynamical masses may be over- or under-estimated due to the dependence on viewing angle, and hence the uncertainties on these mass ratios are somewhat larger than the propagated errors stated in Table 2. All measured spatial separations $(\lesssim 30 \mathrm{kpc})$ are well within the range where they would be deemed gravitationally interacting, and the masses of individual components are $\gtrsim 10^{10} M_{\odot}$, much larger than clumps in a single galaxy (Förster-Schreiber et al. 2009). 
Table 2

Merger Characteristics

\begin{tabular}{|c|c|c|c|c|}
\hline Galaxy & $z$ & $\begin{array}{l}\text { Proj. Sep. } \\
(\mathrm{kpc})\end{array}$ & Mass Ratio (gas) & Mass Ratio (dyn) \\
\hline SMM J09431 a,b & 3.35 & $30 \pm 3$ & $0.41 \pm 0.2$ & $\mathrm{n} / \mathrm{a}$ \\
\hline SMM J131201 & 3.41 & $\leqslant 6$ & $\mathrm{n} / \mathrm{a}$ & $\mathrm{n} / \mathrm{a}$ \\
\hline SMM J105141 ${ }^{\mathrm{a}}$ & 1.21 & $4.9 \pm 0.5$ & $0.26 \pm 0.02$ & $>0.98$ \\
\hline HDF $169^{a}$ & 1.22 & $\leqslant 1.5$ & coal. & Coal. \\
\hline HDF $76^{b, c}$ & 2.20 & $\leqslant 2$ & coal. & Coal. \\
\hline $\operatorname{HDF} 242^{\mathrm{a}, \mathrm{b}, \mathrm{c}}$ & 2.49 & $22 \pm 2$ & $0.54 \pm 0.14$ & $0.85 \pm 0.49$ \\
\hline $\mathrm{N} 2850.2^{\mathrm{b}, \mathrm{c}}$ & 2.45 & $\leqslant 3$ & Coal. & Coal. \\
\hline $\mathrm{N} 2850.4^{\mathrm{a}, \mathrm{b}, \mathrm{c}}$ & 2.38 & $3.7 \pm 0.4$ & $0.68 \pm 0.11$ & $<0.44$ \\
\hline SMM J044307 & 2.51 & $\leqslant 4$ & Coal. & Coal. \\
\hline SMM J02399d & 2.80 & $\sim 8$ & $\mathrm{n} / \mathrm{a}$ & $\mathrm{n} / \mathrm{a}$ \\
\hline $\mathrm{HDF} 132^{\mathrm{e}}$ & 2.00 & $\leqslant 3$ & Coal. & Coal. \\
\hline Lockman $38^{\mathrm{e}}$ & 1.52 & $\leqslant 4$ & Coal. & Coal. \\
\hline
\end{tabular}

Notes. Projected separations and mass ratios. Coal. denotes coalesced system. The distribution of projected separations is also plotted in Figure 6. For SMM J09431, the CO(6-5) data presented in this work were used to derive the gas mass ratio. For SMM J02399, no information on dynamical or gas masses of the subcomponents was available in the literature. As can be seen, the mass ratios are all consistent with major (mass ratio 1:3 or larger) mergers, within the error ranges.

a This work.

b Tacconi et al. (2006).

c Tacconi et al. (2008).

d Ivison et al. (2010).

e Bothwell et al. (2010).

We furthermore note that, as Bothwell et al. (2010) outline, one can also make a strong case for Lockman 38 and HDF 132 being mergers; they show clear indication of two kinematically distinct components, which are however not individually spatially resolved. Bothwell et al. (2010) calculate the mass ratios of the two subcomponents to be $0.24 \pm 0.18$ and $0.68 \pm 0.14$, respectively. However, since these two systems cannot unambiguously be classified as mergers, we do not include them in Table 2.

\section{INTERPRETATION AND DISCUSSION}

\subsection{Sizes}

Size measurements of SMGs based on CO interferometric and $\mathrm{mm}$-continuum data have indicated that these galaxies are comparatively compact, with median diameter $\leqslant 4 \mathrm{kpc}$ (FWHM of Gaussian fitted to source in $u v$-plane; Tacconi et al. 2006). On the other hand, based on the diameter at the $3 \sigma$ detection level of MERLIN and Very Large Array (VLA) radio observations, Chapman et al. (2004) find the star formation to be "occurring on 10 kpc scales." However, Tacconi et al. (2006) and Biggs \& Ivison (2008) point out that the size measurements from the MERLIN and VLA data are consistent with the CO size, when a comparable method (deconvolved major axes of Gaussians fitted to the emission) is applied. They find a median diameter of $\sim 5 \mathrm{kpc}$ for their sample. We can compare radio and $\mathrm{CO}$ size measurements individually for two sources in our sample. For SMM J105141, Biggs \& Ivison (2008) measure an overall radio size of $0 . ' 61 \times 0.44$, or $5.1 \mathrm{kpc} \times 3.7 \mathrm{kpc}$; we measure a $\mathrm{CO}(4-3) \mathrm{FWHM}$ of $3.5 \mathrm{kpc}$ for the main component of this two-galaxy system. For HDF 169, we find a CO(4-3) FWHM of $4.3 \mathrm{kpc}$ and measure $4.5 \mathrm{kpc}$ from an unpublished MERLIN radio map. Thus, there appears to be good agreement between $\mathrm{CO}(4-3)$ and radio extents in these two cases. Younger et al. $(2008,2010)$ measure the scale of far-infrared emission of four SMGs to be $\sim 5-8 \mathrm{kpc}$. This is somewhat larger than what we find for the sample discussed here (although Younger et al. 2008 say that their results are "qualitatively consistent with the results of Tacconi et al. 2006"). The most plausible explanation for the difference-besides the possibility that $\mathrm{CO}$ lines and far-IR continuum trace slightly different things-lies in the fact that the Younger et al. $(2008,2010)$ SMGs probe the brightest end of the luminosity distribution, with $S_{870 \mu \mathrm{m}} \gtrsim 15 \mathrm{mJy}$.

For our augmented sample, which contains all SMGs for which subarcsecond resolution interferometric $\mathrm{CO}$ observations are available (which are not strongly lensed), we have measured FWHM sizes for 11 galaxies (measuring each galaxy

Table 3

Mass Measurements

\begin{tabular}{lccccc}
\hline \hline \multicolumn{1}{c}{ Galaxy } & $\begin{array}{c}M_{\text {gas }} \\
\left(10^{10} M_{\odot}\right)\end{array}$ & $\begin{array}{c}M_{\text {dyn }} \\
\left(10^{10} M_{\odot}\right)\end{array}$ & $\begin{array}{c}M_{\star}{ }^{\mathrm{a}} \\
\left(10^{10} M_{\odot}\right)\end{array}$ & $\begin{array}{c}M_{\star}{ }^{\mathrm{b}} \\
\left(10^{10} M_{\odot}\right)\end{array}$ & $\begin{array}{c}M_{\star}{ }^{\mathrm{c}} \\
\left(10^{10} M_{\odot}\right)\end{array}$ \\
\hline SMM J131201 & $18.0 \pm 9.4$ & $95.0 \pm 23.8$ & $\mathrm{n} / \mathrm{a}$ & $10.2_{-3.3}^{+4.9}$ & 13.5 \\
HDF 169 & $7.5 \pm 5.0$ & $11.2 \pm 2.5$ & $\mathrm{n} / \mathrm{a}$ & $3.2_{-0.8}^{+1.1}$ & 26.9 \\
HDF 76 & $6.6 \pm 1.6$ & $9.6 \pm 4.4$ & $12_{-3}^{+4}$ & $10.2_{-5.3}^{+11.1}$ & 28.2 \\
HDF 242 (SW + NE) & $5.3 \pm 1.3$ & $27.8 \pm 7.4$ & $12_{-4.5}^{+2.5}$ & $3.7_{-1.0}^{+1.4}$ & 52.4 \\
N2850.2 & $10.1 \pm 3.2$ & $14.0 \pm 8.8$ & $25 \pm 7.5$ & $3.2_{-0.9}^{+1.3}$ & 34.6 \\
N2850.4 (SW + NE) & $12.3 \pm 4.0$ & $35.0 \pm 17.9$ & $23_{-7}^{+11}$ & $17.4_{-7.4}^{+12.8}$ & 21.9 \\
HDF 132 & $3.5 \pm 1.1$ & $21.8 \pm 1.8$ & $\mathrm{n} / \mathrm{a}$ & $2.09_{-0.6}^{+0.9}$ & 29.5 \\
SMM J16371+4053 & $5.3 \pm 1.1$ & $\mathrm{n} / \mathrm{a}$ & $\mathrm{n} / \mathrm{a}$ & $4.6_{-1.3}^{+1.7}$ & 47.8 \\
SMM J22174+0015 & $6.7 \pm 1.7$ & $\mathrm{n} / \mathrm{a}$ & $\mathrm{n} / \mathrm{a}$ & $3.4_{-1.8}^{+3.7}$ & 13.8 \\
\hline
\end{tabular}

Notes. Stellar, gas, and dynamical mass measurements for the seven galaxies which are contained both in our sample and those of Michałowski et al. (2010) and Hainline (2008), and two additional galaxies contained in the latter two samples for which we calculate gas masses using fluxes measured by Greve et al. (2005). Michałowski et al. (2010) do not quote uncertainties for their stellar masses. Stellar masses were corrected for a Chabrier (2003) IMF. Binaries are treated as one system. Where multiple transitions were observed, dynamical and gas masses are averages of those derived for the different transitions, see Table 1. As can be seen, the stellar masses derived by Michałowski et al. (2010) are significantly larger than those of Hainline (2008), with only the latter being in good agreement with our gas and dynamical mass measurements.

a Tacconi et al. (2008).

${ }^{\mathrm{b}}$ Hainline (2008).

c Michałowski et al. (2010). 
individually in the case of wide binaries). We find average and median FWHMs of 4.5 and $4.3 \mathrm{kpc}$, with a $1 \sigma$ distribution of $2.8 \mathrm{kpc}$, slightly larger than the results of Tacconi et al. (2006). We are unable to identify a plausible explanation for this, other than small number statistics (Tacconi et al. 2006 measure sizes for five systems, and upper limits for a further four; here, we have size measurements for 12 systems). We also obtain upper limits of 3.4, 6.5, and $2.8 \mathrm{kpc}$ for three other sources (Table 1).

We note that here we are collating measurements from a range of $\mathrm{CO}$ transitions, from (7-6) to (1-0), which may trace physically different environments. As Tacconi et al. (2008) elaborate, transitions up to $J \approx 5-6$ should all provide reasonable tracers of the overall molecular gas. However, the extent to which, say, $\mathrm{CO}(2-1)$ and $\mathrm{CO}(6-5)$ trace the same gas is still ongoing (see also Weiss 2007; Narayanan et al. 2010b). That the $\mathrm{CO}(7-6)$ may trace denser gas might explain the comparatively small source sizes for N2850.2 and N2850.4, and hence be at least partially responsible for the small dynamical masses we derive for these two galaxies (see also the discussion in Danielson et al. 2010). In the one case, where we have a size measurement from two different transitions (SMM J09431 SW; $J=4 \& J=6$ ), the two numbers are consistent within the uncertainties. Restricting to size measurements from $J \leqslant 6$ observations yields average and median FWHMs of 5.2 and $5.0 \mathrm{kpc}$, with a $1 \sigma$ distribution of $2.6 \mathrm{kpc}$.

Thus, both radio and $\mathrm{CO}$ measurements are consistent with SMGs having FWHM sizes of $\sim 5 \mathrm{kpc}$.

\subsection{Stellar Mass Measurements and Their Implications for Cosmological Models}

In this section, we use our dynamical and gas masses to test stellar mass estimates. This is motivated by the large systematic uncertainties associated with stellar mass measurements, and the implications that a reliable determination of the stellar mass has both for a critical assessment of competing cosmological models of SMGs, as well as for the co-evolution of central black holes and their hosts (e.g., Hainline et al. 2010).

\subsubsection{Stellar Mass Measurements}

Hainline (2008; see also Hainline et al. 2010) derived the stellar masses of a large sample of SMGs, employing Spitzer observations to cover a significantly larger spectral range than earlier attempts (e.g., Smail et al. 2004). The Hainline (2008) data set was also used by Michałowski et al. (2010) to derive stellar masses. But despite using an identical observational data base, their results are very different: Hainline (2008) find a median stellar mass of $(6.3-6.9) \times 10^{10} M_{\odot}$, whereas Michałowski et al. (2010) arrive at a median of $3.5 \times 10^{11} \mathrm{M}_{\odot}$. Therefore, testing these results is essential and the next key step toward deriving the stellar mass content of SMGs reliably.

Table 3 brings our dynamical and gas masses together with the stellar masses from Hainline (2008) and Michałowski et al. (2010) for the seven systems in common, and includes gas masses for two more galaxies based on flux measurements of Greve et al. (2005). We also show the stellar masses derived by Tacconi et al. (2008). As noted in Section 3.1, our dynamical masses may be underestimated due to undetected faint extended flux leading to somewhat smaller $\mathrm{HWHM}_{u v}$. However, since this effect would be small and the gas masses also correspondingly larger, this is very unlikely to qualitatively impact our conclusions here. We furthermore note that we have neglected both dust and dark matter in our mass budget. Dust is unlikely to contribute more than a few percent, and Genzel

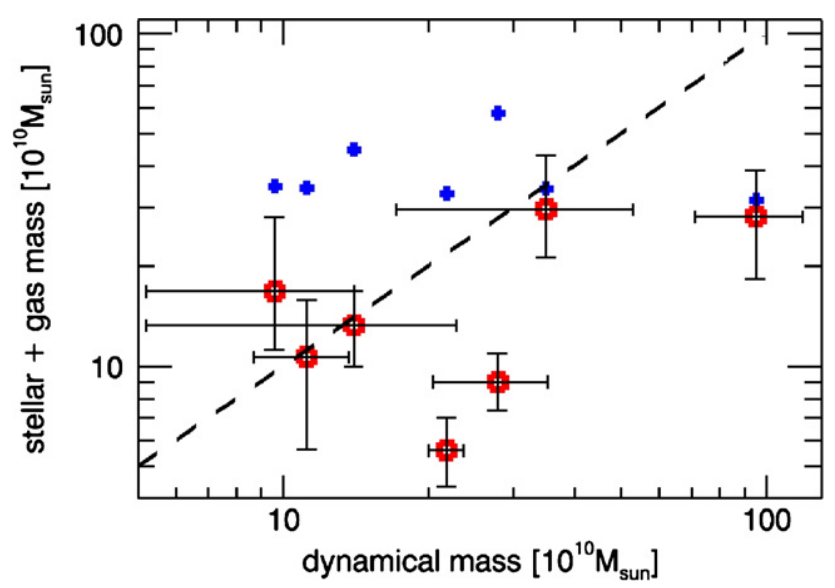

Figure 5. Plot of $\left(M_{\text {gas }}+M_{\star}\right)$ vs. $M_{\text {dyn }}$ for the seven galaxies for which all three mass estimates are available (Table 3 ). Blue crosses denote Michałowski et al. (2010) stellar masses, red squares those of Hainline (2008); both corrected for a Chabrier (2003) IMF. Michałowski et al. (2010) do not quote uncertainties for their stellar masses. The dashed line demarcates $M_{\mathrm{gas}}+M_{\star}=M_{\mathrm{dyn}}$; in order for the mass estimates to be physically realistic, galaxies should lie on or below this line. Within the errors, the Hainline (2008) stellar masses pass this test, whereas those of Michałowski et al. (2010) do not. This constitutes strong support for the Hainline (2008) results.

(A color version of this figure is available in the online journal.)

et al. (2008) find the dark matter content in the central few kpc of high- $z$ galaxies to be $10 \%-20 \%$. Thus, whilst not negligible, these will not have a significant impact on the mass budget, and we have opted not to include it here for the sake of simplicity. We have converted the stellar mass estimates from a Salpeter (1955) to a Chabrier (2003) initial mass function (IMF) by reducing them by a factor of 1.7 (Hainline 2008). This is supported by Bastian et al. (2010), who find no conclusive evidence that an IMF different from the locally applicable Chabrier (2003) or Kroupa (2001) forms should be in place at high redshifts. Cappellari et al. (2009) also find that a Chabrier (2003) instead of a Salpeter (1955) IMF is required to bring the stellar masses derived for a sample of $z \sim 2$ early-type galaxies via SED modeling in agreement with their dynamical masses calculated from Jeans models.

We find that $\left(M_{\text {gas }}+M_{\star}\right) / M_{\text {dyn }}$ for the seven galaxies for which all three mass estimates are available has an average (median) of 0.8 (0.9) for the adjusted Hainline (2008) stellar masses (with a $1 \sigma$ distribution of 0.5 ), whereas for the adjusted Michałowski et al. (2010) masses, the average (median) is 2.1 (2.1), with a $1 \sigma$ distribution of 1.2. We conclude that the Hainline (2008) stellar masses, adjusted to a Chabrier (2003) IMF, are consistent with our independent mass measurements (Figure 5).

\subsubsection{Implications for Cosmological Models}

Two different theoretical approaches to reproduce the submillimeter galaxy population in simulations are currently being pursued. In semi-analytic models (e.g., Swinbank et al. 2008), the high submillimeter luminosities are achieved through bursts of star formation induced in gas-rich major mergers (although disk instabilities may also occur; Bower et al. 2006), whereas numerical simulations have also invoked "cold accretion flows." In the latter, SMGs are assumed to be massive galaxies sitting at the centers of large potential wells, constantly forming stars at high rates sustained by smooth infall and accretion of gas-rich satellites (e.g., Davé et al. 2010).

Both models have shortcomings (such as cold accretion flows being unable to explain the short SMG lifetimes; Tacconi et al. 
2008) and it is likely that both models are fundamentally limited by our current understanding of the role of gas physics inside the haloes. As Benson \& Bowers (2010) show, implementation of cold mode accretion into semi-analytic models does not translate into a significant difference in cosmic star formation history compared to previous models; this may indicate that a more crucial aspect is how the stability of the cold gas inside the haloes is modeled. Equally, the assumptions underlying the numerical simulations have not been fully tested as it is computationally challenging to run large numbers of galaxies in these models to $z=0$ to compare their properties to the observations of local galaxy populations (e.g., luminosity function, color-luminosity relation) to confirm that they reproduce these. Clearly, a detailed discussion of this is beyond the scope of this paper. Instead, we will here look at the stellar masses predicted by the current implementations of each approach, and how these compare to the adjusted Hainline (2008) masses.

Currently, both modeling approaches have the difficulty that if the number density of SMGs is matched, then with a standard IMF, they underpredict the luminosities/SFRs and vice versa (Davé et al. 2010). This may be due to systematics in the simulations (Swinbank et al. 2008) or the inferred properties of observed SMGs (Davé et al. 2010). A key difference between the models is the stellar mass required to match the observed number density. In the cold accretion flow models, SMGs are the most massive galaxies and have a median stellar mass of $2.7 \times 10^{11} M_{\odot}$ (Davé et al. 2010). In the semi-analytic models, the declining stellar mass function, major merger rate, and brief SMG phase push the predicted stellar mass down, and the median is an order of magnitude lower at $\sim 2.1_{-1.0}^{+3.0} \times 10^{10} M_{\odot}$ (Swinbank et al. 2008). In Section 4.2.1, we showed that it is the lower stellar masses, calculated by Hainline (2008) and corrected to a Chabrier (2003) IMF, that are physically more plausible for SMGs. The adjusted median stellar mass for their 64 SMGs is $3.9 \times 10^{10} M_{\odot}$, consistent with what is required by semi-analytic models. We therefore conclude that, although the associated uncertainties are certainly non-negligible, SMG stellar mass measurements strongly disfavor cold accretion flow models, and are compatible with hierarchical merging models.

\subsection{What Triggers the Exceptional Luminosity of SMGs?}

It has previously been conjectured, based primarily on radio and rest-frame UV morphologies, that the high luminosities of SMGs are triggered by merger activity. Our analysis in Section 3 of the $\mathrm{CO}$ emission shows convincing evidence that at least a majority of our 12 SMGs are indeed mergers. In particular, we have found that in all binary SMGs for which we could derive mass ratios, the merger partners are near-equal in mass, i.e., they are major mergers. While it is thus beyond doubt that some SMGs are triggered by major mergers, the question of the nature of the compact, non-binary SMGs comprising a significant fraction of the overall SMG sample is yet to be settled conclusively-are they end-stage major mergers, or isolated disk galaxies?

In the following, we will develop the argument that they mostly are end-stage major mergers, since a major merger will be detectable as an SMG for a significant amount of time spent in the "final coalescence" phase-and thus that the large fraction of observed binary SMGs implies a correspondingly large number of single SMGs, which are coalesced major mergers.

An illustrative comparison can be made with local ULIRGs, known to be exclusively major mergers. As Figure 5 of Veilleux et al. (2002) shows, $\sim 64 \%$ of local ULIRGs are either coalesced,

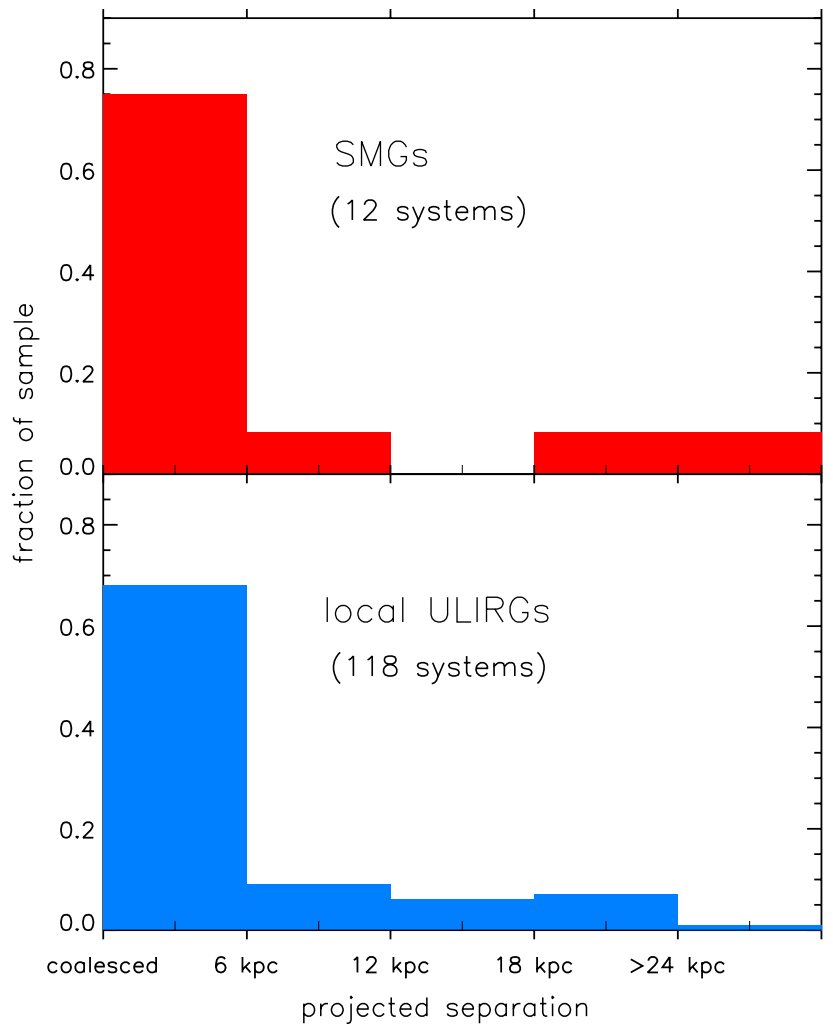

Figure 6. Distribution of projected separations. Red: SMGs, this work, 12 systems. Blue: local ULIRGs, Veilleux et al. (2002), 118 systems. Note that the last bin contains all systems with projected separations larger than $24 \mathrm{kpc}$. We note that for the SMGs, of the nine systems in the first bin (projected separation smaller than $6 \mathrm{kpc}$ ), seven are either poorly resolved or coalesced, and two are clearly recognizable as close binaries; the SMG sample thus divides into five well-resolved binary systems and seven which are either poorly resolved or clearly compact, rotating systems. It should be noted that $\sim 64 \%$ of local ULIRGs have projected separations $\leqslant 2 \mathrm{kpc}$; however, due to the larger upper limits of some of the SMGs, and the increased uncertainties of a finer resolution (due to our small sample size), we have opted to use a coarser resolution. It is evident that SMGs and local ULIRGs display a very similar distribution of projected separations.

(A color version of this figure is available in the online journal.)

or have projected nuclear separations $\leqslant 2 \mathrm{kpc}$. This is what is expected from merger simulations (Mihos \& Hernquist 1996; Springel et al. 2005), which show that a first, minor starburst is triggered upon the first encounter, and the most prodigious star formation rates are only achieved during the final coalescence of the two galaxies, when tidal torquing compresses gas into the center and leads to a $\sim \mathrm{kpc}$-scale central starburst. Could it be that the SMG selection criterion selects a similar class of galaxies, at high redshift, and thus that the large fraction of compact SMGs in our sample is naturally explained as being those end-stage, coalesced major merger products? Indeed, as Figure 6 shows, local ULIRGs and our SMG sample show a strikingly similar distribution of projected separations (here, we have chosen to adopt a coarser bin size than Veilleux et al. 2002 adopted, due to the comparatively small SMG sample size and the relatively large upper limits on some SMG sizes-we note that most of the local ULIRGs in the $\leqslant 6 \mathrm{kpc}$ bin have projected separations $\leqslant 2 \mathrm{kpc}$ ). However, a direct comparison of the two samples is difficult. This is because $L_{\mathrm{IR}}$ and $S_{850 \mu \mathrm{m}}$ may not always be correlated during a major merger, due to the way the IR SED changes with dust temperature at these redshifts-for a given $L_{\mathrm{IR}}, S_{850 \mu \mathrm{m}}$ decreases with increasing dust temperature. This results in a cool dust bias in the SMG sample, illustrated by 
Figure 4 of Casey et al. (2009a), and borne out by observations (Magnelli et al. 2010). This is important, since it may affect which merger stages are sampled by $L_{\mathrm{IR}}$ and $S_{850 \mu \mathrm{m}}$ selection, respectively. Both observations and simulations indicate that dust temperatures increase toward the final stages of a merger, suggesting that SMGs should sample earlier merger stages than ULIRGs. Does this imply that the observed ratio of binary and single SMGs is reconcilable with the single SMGs being isolated disk galaxies, rather than end-stage major merger products as suggested by the comparison with local ULIRGs? In order to answer this, we need to quantify the impact of the SMG cool dust bias on the merger stage sampling function.

Veilleux et al. (2002) use the ratio of IRAS $25 \mu \mathrm{m}$ and $60 \mu \mathrm{m}$ flux as a proxy for dust temperature, and find that local ULIRGs with $f_{25} / f_{60} \geqslant 0.2$ ("warm ULIRGs") are only found among system with nuclear separations below $10 \mathrm{kpc}$ (no trend is evident for smaller $f_{25} / f_{60}$, i.e., cooler systems; their Figure 6). However, the percentage of systems with $f_{25} / f_{60} \geqslant 0.2$ is constant for all nuclear separations $\leqslant 10 \mathrm{kpc}$. More importantly, only 14 out of 118 systems in their sample have $f_{25} / f_{60} \geqslant 0.2$-i.e., less than $10 \%$ of ULIRGs are affected by this bias!

Turning to simulations, Figures 3 and 4 of Narayanan et al. (2010b) and Figure 1 of Narayanan et al. (2010a) show the evolution of the star formation rate, bolometric luminosity, and $S_{850 \mu \mathrm{m}}$ during simulated gas-rich major mergers. These show that after the peak in star formation rate during final coalescence, $S_{850 \mu \mathrm{m}}$ appears to drop off more sharply than $L_{\mathrm{bol}}$, most likely due to an increasing AGN contribution effecting an increase in the dust temperature. However, variations between different simulations make it quite difficult to quantify the time a merger has both high bolometric luminosity and low $S_{850 \mu \mathrm{m}}$ (D. Narayanan 2010, private communication).

Another way to tackle this question may be to compare the AGN luminosity contributions in local ULIRGs and SMGs, since from the above we would expect SMGs to be biased against the merger phases with strong AGN contributions. Veilleux et al. (2009) find local ULIRG luminosities to have $\approx 39 \%$ AGN contribution; estimates for SMGs are more uncertain, but span a range of 8\%-35\% (Swinbank et al. 2004; Alexander et al. 2005, 2008; Lutz et al. 2005; Valiante et al. 2007; Pope et al. 2008; Menéndez-Delmestre et al. 2009). This seems to confirm a significant bias-however, one must bear in mind the differences between high- $z$ and local galaxies; their absolute AGN luminosities should be comparable (similar black hole masses), but the stellar luminosities are factors of several larger in high- $z$ galaxies (due to the larger gas fractions; Tacconi et al. 2010; Daddi et al. 2010), and we therefore expect significantly smaller relative AGN contributions in high- $z$ galaxies. Lower relative AGN contributions in SMGs compared to local ULIRGs are therefore not necessarily a result of a merger stage bias.

The cool dust bias of the $S_{850 \mu \mathrm{m}}$ criterion implies that there should be a population of hot dust, ultra-/hyperluminous high- $z$ galaxies which are not detected as SMGs. And indeed, Casey et al. (2009a) through $70 \mu \mathrm{m}$ selection find a population of $z>1$ galaxies with an average dust temperature of $52 \mathrm{~K}$, which are not detected at $850 \mu \mathrm{m}$. These systems have a mean far-infrared luminosity of $1.9 \times 10^{12} L_{\odot}$, only slightly lower than that of SMGs, and thus it is plausible that they constitute the hot dust extension of the SMG sample. Although uncertain, the number density of these galaxies is estimated to be approximately five times lower than that of SMGs-implying that, if indeed all these systems are major mergers, a high- $z$ major merger may be undetectable as an SMG for approximately 1/6th of its lifetime; or $\approx 25 \%$ of the time spent in the "final coalescence" phase, if all these hot dust systems are in that stage (as one would expect, based on the arguments above). This is larger than, but consistent with, the $\sim 10 \%$ of local ULIRGs showing a dust temperature-merger stage correlation.

It is clear that the merger stage sampling bias of $S_{850 \mu \mathrm{m}}$ versus $L_{\mathrm{IR}}$ selection is difficult to quantify. We can only say with confidence that an $S_{850 \mu \mathrm{m}}$ sampling of a major merger will likely result in a somewhat smaller fraction of coalesced systems, but it certainly will not exclude all of them. Local major mergers, selected via $L_{\mathrm{IR}}$, consist $\sim 64 \%$ of systems with projected nuclear separations $\leqslant 2 \mathrm{kpc}$ (Veilleux et al. 2002); our sample of 12 SMGs contains 7 sources which are either poorly resolved or clearly single galaxies (Table 2). If we conservatively assume that a major merger is undetectable as an SMG for 50\% $(30 \%)$ of the time spent in the "final coalescence" stage, this implies that we would expect the fraction of coalesced systems in the sample to decrease from $\sim 64 \%$ to about $32 \%(45 \%)$. Thus, at most three (one) out of the seven compact/coalesced sources in our sample could plausibly be isolated disk galaxies, rather than end-stage major mergers.

This corroborates the argument made by Tacconi et al. (2006) that the extreme surface densities of compact SMGs can plausibly only be achieved during a major merger, and is further supported by our results of Section 4.2.2, where we show that our confirmation of the Hainline (2008) stellar mass estimates agrees with cosmological models in which SMGs result from major mergers.

These three independent arguments all point in one direction, namely that most, if not all, SMGs are major mergers.

\section{SUMMARY AND CONCLUSIONS}

Our investigation of 12 subarcsecond resolution interferometric CO line observations of SMGs lends strong support to a merger origin of a large majority of this class of galaxies.

1. Five of our systems consist of two spatially distinct galaxies, and the remaining seven have either disturbed morphologies typical for advanced, pre-coalescence mergers or are compact, dense galaxies which plausibly are late-stage, coalesced mergers.

2. All four systems consisting of two resolved galaxies in which the masses of the two separate components could be derived, have mass ratios of 1:3 or closer, within the errors. This demonstrates that they are major mergers.

3. Our gas and dynamical mass estimates are physically consistent with the lower end of the range of stellar masses in the literature, those of Hainline (2008) adjusted to a Chabrier (2003) IMF. This supports hierarchical merger models, whereas it disfavors cold accretion flow models of SMG formation.

4. The majority ( $\sim 64 \%$ ) of local infrared-luminosity-selected major mergers (ULIRGs) are coalesced or have nuclear separations $\leqslant 2 \mathrm{kpc}$. This suggests that the large fraction of binary SMGs in our sample also implies a major merger origin for at least some of the compact/coalesced SMGs. Due to the uncertain difference in merger stage sampling of $L_{\mathrm{IR}} \geqslant 10^{12} L_{\odot}$ and $S_{850 \mu \mathrm{m}} \geqslant 5 \mathrm{mJy}$ selection, we can only estimate the corresponding ratio of binary/coalesced mergers expected in our SMG sample. However, even very conservative assumptions indicate that at most three of the seven compact/coalesced sources in our sample could 
plausibly be isolated disk galaxies, rather than end-stage major mergers.

5. SMG size measurements (Gaussian FWHM) from radio and CO fluxes are both consistent with $\sim 5 \mathrm{kpc}$ diameters (somewhat larger than the results of Tacconi et al. 2006-most plausibly due to small number statistics). These sizes are in agreement with work by Menéndez-Delmestre et al. (2009) and Hainline et al. (2009), who find that the star formation in SMGs is more extended than the kpc-scale central starburst in local ULIRGs. This is consistent with what is expected from theoretical predictions and simulations of mergers with higher gas fractions (e.g., Mihos 1999; Narayanan et al. 2010b).

Theory and observations are only beginning to uncover how differently galaxy evolution proceeds at high redshifts, with the "cold accretion flow" framework successfully addressing many of the shortcomings of the hierarchical merging scenario. Particularly in the lower-luminosity tail of the high- $z$ galaxy population, future observational and theoretical efforts are clearly necessary to fully clarify the picture. However, the evidence presented here overwhelmingly supports a framework in which the most luminous $z \sim 2$ galaxies are powered by major mergers.

We would like to thank the anonymous referee for a thoughtful and thorough reading of the manuscript, which helped to improve and clarify the paper. H.E. would like to thank Desika Narayanan for a helpful discussion of his simulations and the merger evolution of $S_{850 \mu \mathrm{m}}$ versus $L_{\mathrm{IR}}$. We thank the staff of the IRAM observatory for their support of this program. The Dark Cosmology Centre is funded by DNRF. T.R.G. acknowledges support from IDA. I.R.S. acknowledges support from STFC.

\section{REFERENCES}

Alexander, D. M., Bauer, F. E., Chapman, S. C., Smail, I., Blain, A. W., Brandt, W. N., \& Ivison, R. J. 2005, ApJ, 632, 736

Alexander, D. M., et al. 2008, AJ, 135, 1968

Bastian, N., Covey, K. R., \& Meyer, M. R. 2010, ARA\&A, 48, 339

Benson, A. J., \& Bower, R. 2010, arXiv:1004.1162

Biggs, A. D., \& Ivison, R. J. 2008, MNRAS, 385, 893

Blain, A. W., et al. 2002, Phys. Rep., 369, 111

Borys, C., et al. 2005, ApJ, 635, 853

Bothwell, M. S., et al. 2010, MNRAS, 405, 219

Bower, R. G., Benson, A. J., Malbon, R., Helly, J. C., Frenk, C. S., Baugh, C. M., Cole, S., \& Lacey, C. G. 2006, MNRAS, 370, 645

Cappellari, M., et al. 2009, ApJ, 704, 34

Casey, C. M., et al. 2009a, MNRAS, 399, 121

Casey, C. M., et al. 2009b, arXiv:0910.5756

Chabrier, G. 2003, ApJ, 586, 133

Chapman, S. C., Blain, A. W., Ivison, R. J., \& Smail, I. 2003, Nature, 422, 695

Chapman, S. C., Blain, A. W., Smail, I., \& Ivison, R. J. 2005, ApJ, 622, 772

Chapman, S. C., Smail, I., Windhorst, R., Muxlow, T., \& Ivison, R. J. 2004, ApJ, 611,732

Conselice, C. J., Rajgor, S., \& Myers, R. 2008, MNRAS, 386, 909

Coppin, K., Halpern, M., Scott, D., Borys, C., \& Chapman, S. C. 2005, MNRAS, 357, 1022

Cowie, L. L., Barger, A. J., \& Kneib, J. P. 2002, AJ, 123, 2197

Daddi, E., et al. 2010, ApJ, 713, 686

Danielson, A. L. R., et al. 2010, arXiv:1008.3183

Dasyra, K., et al. 2008, ApJ, 680, 232

Dasyra, K. M., et al. 2006, ApJ, 651, 835

Davé, R., Finlator, K., Oppenheimer, B. J., Fardal, M., Katz, N., Keres, D., \& Weinberg, D. H. 2010, MNRAS, 404, 1355

Downes, D., \& Solomon, P. M. 1998, ApJ, 507, 615

Downes, D., \& Solomon, P. M. 2003, ApJ, 582, 37

Elmegreen, D. B., Elmegreen, B. G., Ravindranath, S., \& Coe, D. A. 2007, ApJ, 658,763

Förster-Schreiber, N. M., et al. 2006, ApJ, 645, 1062

Förster-Schreiber, N. M., et al. 2009, ApJ, 706, 1364

Frayer, D. T., et al. 2008, ApJ, 680, 21
Genzel, R., Baker, A. J., Tacconi, L. J., Lutz, D., Cox, P., Guilloteau, S., \& Omont, A. 2003, ApJ, 584, 633

Genzel, R., et al. 2006, Nature, 442, 786

Genzel, R., et al. 2008, ApJ, 687, 59

Genzel, R., et al. 2010, MNRAS, 407, 2091

Greve, T. R., Ivison, R. J., Bertoldi, F., Stevens, J. A., Dunlop, J. S., Lutz, D., \& Carilli, C. L. 2004, MNRAS, 354, 779

Greve, T. R., et al. 2005, MNRAS, 359, 1165

Guilloteau, S., et al. 1992, A\&A, 262, 624

Hainline, L. J. 2008, PhD Thesis, California Inst. Technology

Hainline, L. J., Blain, A. W., Greve, T. R., Chapman, S. C., Smail, I., \& Ivison, R. J. 2006, ApJ, 650, 614

Hainline, L. J., Blain, A. W., Smail, I., Alexander, D. M., Armus, L., Chapman, S. C., \& Ivison, R. J. 2010, arXiv:1006.0238

Hainline, L. J., Blain, A. W., Smail, I., Frayer, D. T., Chapman, S. C., Ivison, R. J., \& Alexander, D. M. 2009, ApJ, 699, 1610

Holland, W. S., et al. 1999, MNRAS, 303, 659

Ivison, R. J., Smail, I., Barger, A. J., Kneib, J.-P., Blain, A. W., Owen, F. N., Kerr, T. H., \& Cowie, L. L. 2000, MNRAS, 315, 209

Ivison, R. J., Smail, I., Le Borgne, J.-F., Blain, A. W., Kneib, J.-P., Bezecourt, J., Kerr, T. H., \& Davies, J. K. 1998, MNRAS, 298, 583

Ivison, R., Smail, I., Papadopoulos, P. P., Wold, I., Richard, J., Swinbank, A. M., Kneib, J.-P., \& Owen, F. N. 2010, MNRAS, 404, 198

Ivison, R. J., et al. 2002, MNRAS, 337, 1

Ivison, R. J., et al. 2007, MNRAS, 380, 199

Johansson, P. H., Naab, T., \& Burkert, A. 2009, ApJ, 690, 802

Kreysa, E., et al. 1998, Proc. SPIE, 3357, 319

Kroupa, P. 2001, MNRAS, 322, 231

Lotz, J. M., Madau, P., Giavalisco, M., Primack, J., \& Ferguson, H. C. 2006, ApJ, 636,592

Lutz, D., Yan, L., Armus, L., Helou, G., Tacconi, L. J., Genzel, R., \& Baker, A. J. 2005, ApJ, 632, 13

Magnelli, B., et al. 2010, A\&A, 518, 28

Menéndez-Delmestre, K., et al. 2009, ApJ, 699, 667

Michałowski, M. J., Hjorth, J., \& Watson, D. 2010, A\&A, 514, 67

Mihos, C., \& Hernquist, L. 1996, ApJ, 464, 641

Mihos, J. C. 1995 , ApJ, 438, 75

Mihos, J. C. 1999, Ap\&SS, 266, 195

Narayanan, D., Hayward, C. C., Cox, T. J., Hernquist, L., Jonsson, P., Younger, J. D., \& Groves, B. 2010a, MNRAS, 401, 1613

Narayanan, D., et al. 2009, MNRAS, 400, 1919

Narayanan, D., et al. 2010b, MNRAS, 407, 1701

Neri, R., et al. 2003, ApJ, 597, 113

Pei, Y. C., Fall, S. M., \& Hauser, M. G. 1999, ApJ, 522, 604

Pope, A., Borys, C., Scott, D., Conselice, C., Dickinson, M., \& Mobasher, B. 2005, MNRAS, 358, 149

Pope, A., et al. 2008, ApJ, 675, 1171

Puget, J.-L., Abergel, A., Bernard, J.-P., Boulanger, F., Burton, W. B., Desert, F.-X., \& Hartmann, D. 1996, A\&A, 308, 5

Salpeter, E. E. 1955, ApJ, 121, 161

Smail, I., Chapman, S. C., Blain, A. W., \& Ivison, R. J. 2004, ApJ, 616, 71

Smail, I., Ivison, R. J., Blain, A. W., \& Kneib, J.-P. 2002, MNRAS, 331, 495

Smail, I., Smith, G. P., \& Ivison, R. J. 2005, ApJ, 631, 121

Solomon, P. M., Downes, D., Radford, S. J. E., \& Barrett, J. W. 1997, ApJ, 478, 144

Spitzer, L., Jr. 1987, Dynamics and Evolution of Globular Clusters (Princeton, NJ: Princeton Univ. Press)

Springel, V., Di Matteo, T., \& Hernquist, L. 2005, MNRAS, 361, 776

Swinbank, A. M., Smail, I., Chapman, S. C., Blain, A. W., Ivison, R. J., \& Keel, W. C. 2004, ApJ, 617, 64

Swinbank, A. M., Chapman, S. C., Smail, I., Lindner, C., Borys, C., Blain, A. W., Ivison, R. J., \& Lewis, G. F. 2006, MNRAS, 371, 465

Swinbank, A. M., et al. 2008, MNRAS, 391, 420

Swinbank, A. M., et al. 2010, MNRAS, 405, 234

Tacconi, L. J., et al. 2006, ApJ, 640, 228

Tacconi, L. J., et al. 2008, ApJ, 680, 246

Tacconi, L. J., et al. 2010, Nature, 463, 781

Valiante, E., Lutz, D., Sturm, E., Genzel, R., Tacconi, L. J., Lehnert, M. D., \& Baker, A. J. 2007, ApJ, 660, 1060

Veilleux, S., Kim, D.-C., \& Sanders, D. B. 2002, ApJS, 143, 315

Veilleux, S., et al. 2009, ApJS, 182, 628

Weiss, A. 2007, in ASP Conf. Ser. 375, From z-Machines to ALMA: Submillimetre-spectroscopy of Galaxies, ed. A. J. Baker et al. (San Francisco, CA: ASP), 25

Weiss, A., et al. 2009, ApJ, 707, 1201

Younger, J. D., et al. 2008, ApJ, 688, 59

Younger, J. D., et al. 2010, MNRAS, 407, 1268 